\title{
DEPRESIÓN MATERNA Y EL CRECIMIENTO Y DESARROLLO DEL NIÑO DE 0 A 12 MESES MOROCOCHA - YAULI LA OROYA 2008*
}

\author{
Yallico Madge, Luz Consuelo , Yallico Madge, María Cleofé² y Palomino Flores, Yely³ \\ Facultad de Enfermería de la Universidad Nacional del Centro del Perú
}

\begin{abstract}
RESUMEN
La madre constituye el ambiente inmediato del niño, cuando este ambiente no es favorable los factores determinantes se convierten en riesgos para el crecimiento y el desarrollo. El objetivo de la investigación fue estudiar la influencia de la Depresión Materna en el Crecimiento y Desarrollo de niños y niñas de 0 a 12 meses del distrito de Morococha. El tamaño muestral fue de 32 madres con sus hijos. Los datos encontrados demostraron que el bienestar y la tranquilidad de la madre influyen en el crecimiento y desarrollo del niño y niña; el éxito en las acciones que el niño realiza en su primer año de vida, acrecentará su placer y las repetirá hasta dominar la conducta especifica en la que tuvo éxito, mientras que abandonará las acciones que llevan al fracaso. En el proceso de crecimiento y desarrollo del niño y niña hay una serie de intercambios entre madre e hijo; cada uno de los cuales influye recíprocamente en el otro. Freud llamó a esta dualidad "Masa de dos". En el estudio el 69\% de las madres estaban pasando por un proceso depresivo, y de los niños y niñas de 0 a 12 meses el $44 \%$ de ellos tenía déficit en el Desarrollo Psicomotor, con respecto al estado nutricional de los niños y niñas el $44 \%$ de ellos tenía el diagnóstico de desnutrición crónica y el 25\% anemia. Por ello después del análisis y de los datos encontrados concluimos en que la depresión materna es un factor de riesgo para el crecimiento y desarrollo de sus hijos.
\end{abstract}

Palabras Clave: depresión materna, desarrollo psicomotor, test de Hamilton, hemocue, desnutrición.

\section{MATERNAL DEPRESSION AND CHILD GROWTH AND DEVELOPMENT FROM 0 TO 12 MONTHS MOROCOCHA - LA OROYA YAULI 2008}

\begin{abstract}
The mother is the child's immediate environment when this environment is not favorable the determinants factors become on risks to growth and development. The research objective was to study the influence of maternal depression on the child Growth and Development of 0 to 12 months age in Morococha district. The sample size was 32 mothers with their children. The found data showed that the mother welfare and tranquility influence the growth and development of the child. The success of the actions the child performs in the first year of life, will increase the pleasure and will repeat them to master the specific conduct that is successful, while abandoning the actions that lead to failure. In the growth and development process of the child, there is a series of exchanges between mother and child, each of them affects each other. Freud called this duality a "mass of two." In the study $69 \%$ of mothers were going through a depressive process, and $44 \%$ of children aged 0 to 12 months are deficient in psychomotor development. Regarding the nutritional status of children, $44 \%$ suffer chronic malnutrition and $25 \%$ have anemia. Therefore we conclude that maternal depression is a risk factor for growth and development of their children.
\end{abstract}

Key words: maternal depression, developmental delay, test in Hamilton, hemocue, malnutrition.

\footnotetext{
* Este trabajo de investigación fue recibido el 20/04/2008 retornado para su revisión el 26/10/2009 y aprobado para su publicación 16/11/2009

1Email:consueloyallico@yahoo.it

2Email:cyallico@yahoo.es

3 Email:yely_vc@yahoo.es
} 


\section{INTRODUCCIÓN}

La mujer tiene el doble de probabilidad en sufrir de depresión que el varón, ya que através de los estudios realizados han demostrado que las hormonas sexuales femeninas predisponen a las mujeres especialmente en el embarazo y la etapa del postparto. Los niños cuyas madres han sufrido de depresión antes o después del parto tienen un crecimiento y desarrollo más lento que los hijos de las mujeres que se encuentran bien psicológicamente. Una vez que ha nacido el bebé, las madres deprimidas no proporcionan a sus hijos los cuidados físicos y psicológicos que requieren ni los estimulan socialmente. La falta de apoyo familiar y de recursos económicos también perjudica la salud de los pequeños. Tanto impacto tiene la depresión materna en el crecimiento y desarrollo de los pequeños que si se consiguiera disminuir el número de madres deprimidas se podría reducir los casos de retraso en el crecimiento y desarrollo de los niños.

\section{MATERIALES Y MÉTODOS}

El estudio es de tipo descriptivo correlacional por su naturaleza ya que busca la relación causa efecto de las variables depresión materna y crecimiento y desarrollo del niño. La población sujeta a la investigación estuvo conformada por 120 madres de niños de 0 a 12 meses distribuida en el distrito de Morococha teniendo en cuenta sus tres sectores geográficos: Morococha Nueva, Morococha Vieja y Pucará.

Los métodos de investigación que se utilizaron fueron el método básico de la ciencia, además del inductivo, deductivo, estadístico y analítico.

La población total de madres con hijos de 0 a 12 meses en dicho distrito es de 120 , por tanto la muestra fue de 32 madres y niños y niñas de 0 a 12 meses, cantidad hallada por muestreo aleatorio simple, modalidad que alcanza mayor rigor científico y garantiza la equiprobabilidad de elección de cualquier elemento y la independencia de selección de cualquier otro.
Se tuvo en cuenta como criterios de inclusión del estudio a madres con niños o niñas de 0 a 12 meses y 29 días de edad. Se excluyo del estudio a las madres que no desearon participar en la investigación.

Las variables centrales del estudio fueron: depresión materna y desarrollo psicomotor.

\section{Técnicas e Instrumentos}

- Test psicométrico de depresión de Hamilton: Creada por Hamilton en 1960, es una escala de autoevaluación que consiste en obtener una medida del estado depresivo. Esta escala a pesar de no ser cronológicamente la primera para evaluar la depresión se ha convertido rápidamente en el término de comparación para todas las demás escalas.

- Evaluación del desarrollo psicomotor: Para la evaluación del desarrollo psicomotor se utilizó la batería de prueba, el instructivo y ficha de registro y protocolo del Test Abreviado de Evaluación del Desarrollo Psicomotor del niño de 0 a 12 meses, que es una simplificación de la Escala de Evaluación del Desarrollo Psicomotor (EEDP) que evalúa las respuestas en las áreas: social, lenguaje, motor y coordinación.

- Evaluación del crecimiento: La evaluación del crecimiento se realizó con la medición del peso y la talla de los niños y niñas. El diagnóstico del estado nutricional se realizó en base a la comparación del peso/ talla según edad con los padrones de referencia de la OMS; clasificando el estado nutricional del menor según se muestra en el siguiente cuadro.

- Nivel de hemoglobina: Para la determinación de la hemoglobina se utilizó el sistema Hemocue, instrumento que permite la medición de la hemoglobina en forma fotométrica. La medición de la hemoglobina en zonas de altura como Morococha donde la presión de $\mathrm{O}_{2}$ es reducida en comparación con las poblaciones que viven a nivel del mar requiere de la aplicación de una fórmula de ajuste que permita obtener el valor exacto y que nos permita sacar un diagnostico preciso, para esto se ha realizado el procedimiento de conversión con las tablas de ajuste del Instituto Nacional de Salud, permitiéndonos obtener valores ajustados al nivel del mar. 


\section{RESULTADOS Y DISCUSIÓN}

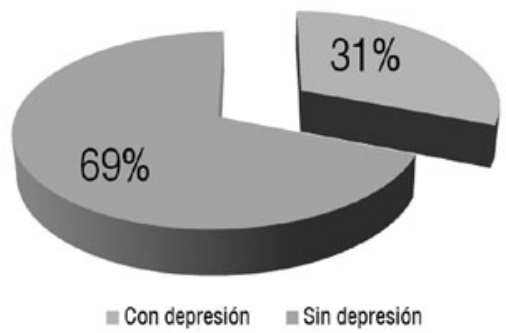

Gráfico 1: Depresión en las madres con niños y niñas de 0 a 12 meses

El $69 \%$ de las madres con niños de 0 a 12 meses del distrito de Morococha sufren de depresión, teniendo en cuenta el porcentaje del total de madres por cada sector. En el $79 \%$ de las madres deprimidas se encuentra como principales características: el humor deprimido, tristeza (melancolía), desesperanza, desamparo, inutilidad y sentimiento de culpa. Al 5\% de estas madres le parece que la vida no vale la pena ser vivida y el $32 \%$ presenta insomnio tardío (Se despierta por las noches, pero se vuelve a dormir).

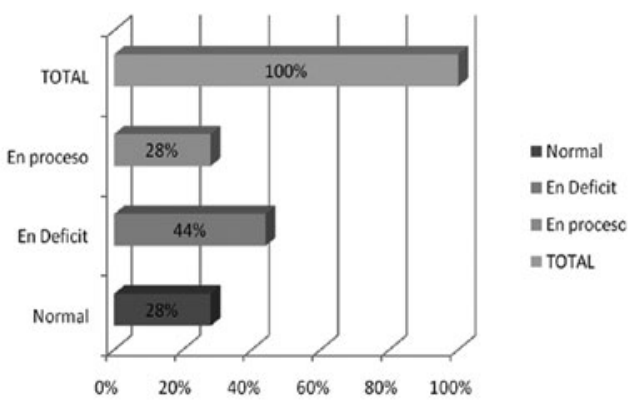

Gráfico 2: Desarrollo psicomotor de los niños de 0 a 12 meses

El 44\% de los niños y niñas de 0 a 12 meses presentan déficit en el Desarrollo Psicomotor; y solo el $28 \%$ ha alcanzado un desarrollo psicomotor óptimo para su edad.

De los niños y niñas con déficit encontramos que el $26 \%$ se encuentra con déficit en el área Social, el 17\% en el área de Lenguaje, el 14\% en el área de Comunicación y un $43 \%$ en el área psicomotora, siendo esta última la mas prevalente en los niños y niñas.

Tabla 1: Relación entre depresión materna, déficit del desarrollo psicomotor, desnutrición crónica y anemia de los niños y madres del distrito de Morococha.

\begin{tabular}{lccccccc}
\hline LUGAR & \multicolumn{2}{c}{ DEFICIT } & \multicolumn{2}{c}{ NORMAL } & \multicolumn{2}{c}{ EN PROCESO } \\
\hline & № & $\%$ & № & $\%$ & № & $\%$ & TOTAL \\
$\begin{array}{c}\text { Morococha } \\
\text { Nueva }\end{array}$ & 3 & $21 \%$ & 2 & $22 \%$ & 1 & $11 \%$ & 6 \\
$\begin{array}{c}\text { Morococha } \\
\text { Vieja }\end{array}$ & 8 & $58 \%$ & 6 & $67 \%$ & 5 & $56 \%$ & 19 \\
Pucará & 3 & $21 \%$ & 1 & $11 \%$ & 3 & $33 \%$ & 7 \\
Total & 14 & $44 \%$ & 9 & $28 \%$ & 9 & $28 \%$ & 32 \\
\hline
\end{tabular}

De los niños evaluados el $44 \%$ de ellos tiene desnutrición crónica y el $38 \%$ se encuentra en riesgo desnutrición crónica. En cuanto a la desnutrición aguda encontramos que el $3 \%$ de los niños y niñas la sufre; $y$, un $32 \%$ de ellos tiene desnutrición global y en riesgo de caer a esta un $9 \%$. Siendo más preocupante el estado de los niños con talla baja.

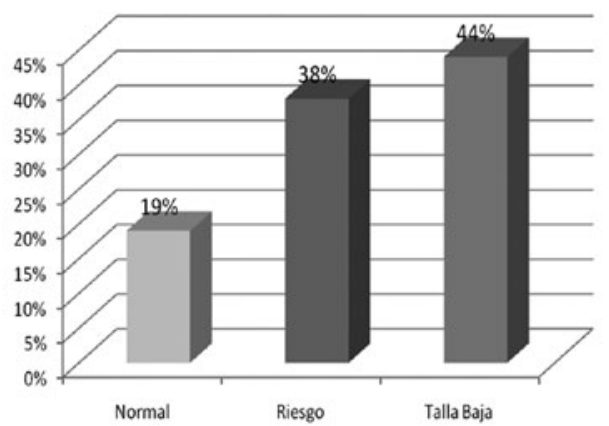

Gráfico 3: Estado nutricional de los niños y niñas de 0 a 12 meses.

También encontramos que el $25 \%$ de niños y niñas sufren de anemia, encontrándose con valores menores a 15.5gr/dl de hemoglobina. Volvemos a encontrar la mayor cantidad de niños con anemia en el sector de Morococha Vieja con un 58\% y en Pucará la menor prevalencia con un $17 \%$.

Con respecto a la relación entre Depresión Materna, déficit del Desarrollo Psicomotor, Desnutrición Crónica y Anemia, hallamos que el $44 \%$ de los niños y niñas de 0 a 12 meses sufren tanto de Desnutrición Crónica como de Déficit en el Desarrollo Psicomotor; encon- 
trándose también este grupo con anemia y teniendo al $69 \%$ de las madres con depresión.

\section{DISCUSIÓN}

La infancia temprana es el periodo de desarrollo más rápido en la vida humana. A pesar de que los niños y niñas se desarrollan individualmente y a su propio ritmo, todos los pasan por secuencias identificables de cambio y desarrollo físico, cognitivo y emocional. El enfoque del Desarrollo Infantil Temprano se basa en el hecho comprobado de que los niños pequeños responden mejor cuando las personas que los cuidan usan técnicas diseñadas específicamente para fomentar y estimular el paso al siguiente nivel de desarrollo.

El crecimiento y desarrollo se presenta en forma acelerada durante el período temprano, en comparación con los años posteriores. Es por esto que si el niño no tiene las condiciones necesarias durante esta etapa, habrá perdido las mejores oportunidades para desarrollar su potencial al máximo. Las múltiples evidencias científicas prueban que los factores propios del ambiente en el cual el niño crece y se desarrolla son iguales o más importantes que los genes. Estos factores incluyen la nutrición, la salud, afecto y la protección contra daños. Entonces el niño depende en gran medida de la situación emocional de la madre que es determinante para propiciar su cuidado adecuado.

En el presente estudio encontramos que el $44 \%$ de los niños tienen desnutrición crónica y déficit en el desarrollo psicomotor y el $75 \%$ sufre de anemia. Teniendo en cuenta los estudios de Field en 1984 y Bettes en 1988, donde compararon las interacciones cara a cara de madres deprimidas y no deprimidas con sus bebes. Las madres deprimidas revelaron menos expresiones faciales positivas, más expresiones negativas, menos vocalizaciones, y miraban y tocaban menos a sus hijos. A su vez los bebés de las madres deprimidas mostraron menos expresiones faciales positivas, más expresiones faciales negativas, menos vocalizaciones, mayor desviación de la mirada, mas protestas y niveles superiores de actividad. La conducta de estos niños indicó que por su frecuente exposición a las conductas deprimidas de sus madres, desarrollaron un estilo deprimido de interacción. Esta conducta del niño se debe al reflejo de la conducta de su madre 0 a la poca atención, cuidado y estimulación que recibe el niño de la madre deprimida.

El bienestar y la tranquilidad de la madre afectan el desarrollo al niño o niña. El placer del bebe se acrecienta aun más cuando la madre participa de sus regocijos. El éxito en las acciones que el niño realiza en su primer año de vida, acrecentará su placer y repetirá hasta dominar la conducta específica que haya tenido éxito, mientras que abandona las acciones que llevan lo llevan al fracaso. En el proceso de crecimiento y desarrollo del niño hay una serie de intercambios entre la madre y el hijo; cada uno de los cuales influye recíprocamente al otro, es así que el desarrollo depende de la nutrición del niño y de la estimulación brindada por la madre. Freud llamo a esta dualidad, una "masa de dos". En el estudio el $69 \%$ de las madres están pasando por un proceso depresivo, experimentando: inseguridad, inestabilidad emocional y temor en la crianza de sus niños, lo que les impide dar un cuidado adecuado a sus niños o niñas.

\section{CONCLUSIONES}

- La depresión materna influye directamente el crecimiento y desarrollo psicomotor del niño.

- La madre constituye el ambiente inmediato del niño, y cuando este ambiente no es favorable los factores determinantes se convierten en riesgos para el crecimiento y el desarrollo. Estos factores tienen un impacto interactivo y acumulativo a través de la edad y el tipo e intensidad del daño varía de acuerdo con el momento en el cual éste se presenta.

- Cada área del desarrollo tiene períodos críticos en diferentes momentos que no pueden ser significantemente modificados o mejorados posteriormente con cambios en el ambiente. Cuando no se completa o se demora el desarrollo de un área específica, las siguientes fases del desarrollo pueden ser impedidas con consecuencias o retrasos que pueden permanecer por el resto de la vida.

${ }^{2}$ Tomado de "El Desarrollo Infantil Temprano, Invirtiendo en las personas" de Mary Eming Young, Banco Mundial 


\section{LITERATURA CITADA}

Kramer, Peter D. 2006. Contra la depresión. Barcelona: Seix Barral.

Jackson, Stanley W. 1986. Historia de la melancolía y la depresión. Madrid: Turner.

Vara Horna, Arístides A. 2006. Aspectos generales de la depresión: Una revisión empírica. Asociación por la Defensa de las Minorías: Lima.

\section{Departamento de Salud e Higiene Mental de} Maryland. 2004. Con colaboración y apoyo de la Administración de Salud Familiar y Administración de Higiene Mental, Departamento de
Salud e Higiene Mental de Maryland: Depresión y madres primerizas.

Ayudo Gutiérrez, J. L. 1980. Trastornos afectivos.

Rivera J. L. y otros 1980. Manual de psiquiatría. Madrid: Karpos.

Williams, JB. August 1988. A Structured Interview Guide for the Hamilton Depression Rating Scale, Archives of General Psychiatry, American Medical Association, Vol. 45, Num. 8, pp. 742-747.

Eming Young Mary. 2003. El Desarrollo Infantil temprano, Lecciones aprendidas, Banco Mundial. 